\title{
Appendix
}

\section{Functional Integration}

The ultimate mathematical step that one has to perform in the problems that we have discussed so far is an average. This is expressed in terms of the N-point correlation function

$$
\begin{aligned}
G_{N}\left(x_{1}, x_{2} \ldots x_{N}\right) & =\left\langle\phi\left(x_{1}\right) \phi\left(x_{2}\right) \ldots \ldots \phi\left(x_{N}\right)\right\rangle \\
& =\frac{1}{Z} \int \mathcal{D}[\phi] \phi\left(x_{1}\right) \phi\left(x_{2}\right) \ldots \ldots \phi\left(x_{N}\right) e^{-\int F d^{D} x}
\end{aligned}
$$

Here we have used a scalar field in defining $G_{N}$ for simplicity but extension to vector fields is quite straightforward. In the above expression, the partition function $Z$ and the free energy $F$ are

$$
\begin{aligned}
Z(h(x)) & =\int \mathcal{D}[\phi] e^{-\int F(h(x)) d^{D} x} \\
F(h(x) & =\frac{1}{2}(\vec{\nabla} \phi)^{2}+\frac{1}{2} m^{2} \phi^{2}+\frac{\lambda}{4 !}\left(\phi^{2}\right)^{2}-h(x) \phi
\end{aligned}
$$

the $h \rightarrow 0$ limits of the above expressions. It follows that

$$
G_{N}\left(x_{1}, x_{2} \ldots x_{N}\right)=\left.\frac{1}{Z} \frac{\delta^{N} Z(h(x))}{\delta h\left(x_{1}\right) \delta h\left(x_{2}\right) \ldots . . \delta h\left(x_{N}\right)}\right|_{h=0}
$$

The integral (A.0.1) can be solved exactly only if $\lambda=0$. For $\lambda \neq 0$ one can make use of perturbation technique to achieve the desired accuracy for small $\lambda$, otherwise one has to fall back on computers for numerical evaluation. 
Here we evaluate $Z$ for $\lambda=0$.

$$
\begin{aligned}
Z & =\int \mathcal{D}[\phi] e^{-\int d^{D} x\left[\frac{1}{2}(\vec{\nabla} \phi)^{2}+\frac{1}{2} m^{2} \phi^{2}\right]} \\
& =\int \mathcal{D}[\phi] e^{-\int d^{D} x \phi\left[-\frac{1}{2} \nabla^{2}+m^{2}\right] \phi} \\
& =\int \mathcal{D}[\phi] e^{-\int d^{D} x \phi \mathcal{B} \phi}
\end{aligned}
$$

where $\mathcal{B}$ represents the operator $-\frac{1}{2} \nabla^{2}+m^{2}$. Every integral, in principle can be thought of as the limit of a sum. Hence to evaluate this integral we replace the continuum by a lattice and at each point $i$ of the lattice we replace $\phi(x)$ by $\phi_{i}$ where the variable $\phi_{i}$ can range from $-\infty$ to $\infty$. Under this transformation

$$
\int d^{D} x \phi \mathcal{B} \phi \rightarrow \sum_{i j} \phi_{i} B_{i j} \phi_{j}
$$

with the operational meaning of $\mathcal{D}[\phi]$ being

$$
\mathcal{D}[\phi]=\Pi_{i=1}^{N} \int_{-\infty}^{\infty} d \phi_{i}
$$

Therefore the discretized version of Eq.(A.0.5) appears as

$$
Z=\Pi_{i=1}^{N} \int_{-\infty}^{\infty} d \phi_{i} e^{-\sum_{j k} \phi_{j} B_{j k} \phi_{k}}
$$

where the matrix $B_{j k}$ is symmetric. Here, antisymmetry would imply vanishing of the sum. This allows for diagonalization by an orthogonal transformation. From now on we will use Einstein's summation convention to imply that repeated indices are summed over. We consider an orthogonal transformation $S$ that diagonalizes $B$ such that

$$
B_{j k}=S_{j m} D_{m n} S_{n k}^{T}
$$

where $D$ is the diagonal matrix

$$
D_{m n}=D_{m n} \delta_{m n} .
$$

Starting from

$$
\begin{aligned}
\phi_{j} B_{j k} \phi_{k} & =\phi_{j} S_{j m} D_{m n} S_{n k}^{T} \phi_{k} \\
& =\psi_{m} D_{m n} \phi_{n}
\end{aligned}
$$


where

$$
\text { and } \quad \begin{aligned}
\phi_{j} S_{j m} & =\psi_{m} \\
\phi_{m} & =S_{m n} \psi_{n}
\end{aligned}
$$

Using the fact that the Jacobian of the transformation is unity, i.e. $\operatorname{Det}(S)=1$, we have

$$
\Pi_{k} d \phi_{k}=\Pi_{k} d \psi_{k}
$$

Taking into account that $S$ is orthogonal, i.e. $S S^{T}=1$ we have

$$
\begin{aligned}
Z & =\Pi_{i=1}^{N} \int_{-\infty}^{\infty} d \psi e^{-\frac{1}{2} D_{j j} \psi_{j}^{2}} \\
& =\left[\int_{-\infty}^{\infty} d \psi e^{-\frac{1}{2} D_{j j} \psi_{j}^{2}}\right]^{N} \\
& =(2 \pi)^{N / 2}\left[\frac{1}{\operatorname{Det}(D)}\right]^{1 / 2} \\
& =(2 \pi)^{N / 2}\left[\frac{1}{\operatorname{Det}(B)}\right]^{1 / 2}
\end{aligned}
$$

It is apparent from the given structure of $F$ that

$$
\left\langle\phi\left(x_{1}\right) \phi\left(x_{2}\right) \ldots \ldots \phi\left(x_{2 m+1}\right)\right\rangle=0
$$

in the limit of $h \rightarrow 0$.

For the two point function we have

$$
\begin{aligned}
{\left[\Pi_{i=1}^{N} \int_{-\infty}^{\infty} d \phi_{i}\right] \phi_{p} \phi_{q} e^{-\frac{1}{2} \phi_{j} B_{j k} \phi_{k}} } & =\left[\Pi_{i=1}^{N} \int_{-\infty}^{\infty} d \phi_{i}\right] \\
\phi_{q} B_{p r}^{-1} \frac{\delta}{\delta \phi_{r}} e^{-\frac{1}{2} \phi_{j} B_{j k} \phi_{k}} & \\
= & {\left[\Pi_{i=1}^{N} \int_{-\infty}^{\infty} d \phi_{i}\right] B_{p r}^{-1} \delta_{r q} e^{-\frac{1}{2} \phi_{j} B_{j k} \phi_{k}} } \\
& =B_{p r}^{-1}(2 \pi)^{N / 2}\left[\frac{1}{\operatorname{Det}(B)}\right]^{1 / 2}
\end{aligned}
$$

where in the penultimate step we have integrated by parts. Hence, we can readily write (by induction)

$$
\left[\Pi_{i=1}^{N} \int_{-\infty}^{\infty} d \phi_{i}\right] \phi_{i_{1}} \phi_{i_{2}} \ldots \ldots \phi_{i_{2 n+1}} e^{-\frac{1}{2} \phi_{j} B_{j k} \phi_{k}}=0
$$


and

$$
\begin{aligned}
& {\left[\Pi_{i=1}^{N} \int_{-\infty}^{\infty} d \phi_{i}\right] \phi_{i_{1}} \phi_{i_{2}} \ldots \ldots \phi_{i_{2 n}} e^{-\frac{1}{2} \phi_{j} B_{j k} \phi_{k}}} \\
& \quad=(2 \pi)^{N / 2}(\operatorname{Det} B)^{-1 / 2}\left[B_{i_{1}, i_{2}}^{-1} B_{i_{3}, i_{4}}^{-1} \ldots B_{i_{2 n-1}, i_{2 n}}^{-1}+\text { all possible pairings }\right]
\end{aligned}
$$

Thus,

$$
\left\langle\phi_{i_{1}} \phi_{i_{2}} \ldots \ldots \phi_{i_{2 n}}\right\rangle=B_{i_{1}, i_{2}}^{-1} B_{i_{3}, i_{4}}^{-1} \ldots . B_{i_{2 n-1}, i_{2 n}}^{-1}+\text { all possible pairings }
$$

This is the Wick's theorem in the parlance of functional integration.

\section{Appendix.1 Gaussian Theory}

In this theory $\lambda=0$. To calculate the correlation function, we go to momentum space which facilitates calculation. We define Fourier transform pair as

$$
\begin{aligned}
& \phi(x)=\int \frac{d^{D} p}{(2 \pi)^{D}} \tilde{\phi}(p) e^{-i \vec{p} \cdot \vec{x}} \\
& \tilde{\phi}(p)=\int d^{D} x \phi(x) e^{i \vec{p} \cdot \vec{x}}
\end{aligned}
$$

which allows us to write

$$
\begin{aligned}
\int d^{D} x \phi(x)\left[-\nabla^{2}+m^{2}\right] \phi(x)= & \int d^{D} x \int \frac{d^{D} p}{(2 \pi)^{D}} \tilde{\phi}(p) e^{-i \vec{p} \cdot \vec{x}}\left[-\nabla^{2}+m^{2}\right] \\
& \times \int \frac{d^{D} q}{(2 \pi)^{D}} \tilde{\phi}(q) e^{-i \vec{q} \cdot \vec{x}} \\
= & \int \frac{d^{D} p}{(2 \pi)^{D}} \tilde{\phi}(p)\left[p^{2}+m^{2}\right] \tilde{\phi}(-p)
\end{aligned}
$$

The operator $\mathcal{B}$ is thus diagonal in momentum space and hence $\mathcal{B}^{-1}=\left(p^{2}+m^{2}\right)^{-1}$. The Green's function $G_{0}(p)$ of the Gaussian theory is thus

$$
G_{0}(p)=\frac{1}{p^{2}+m^{2}}
$$

Higher order Green's functions can also be found from the Eq.(A.0.16).

We now try to find the susceptibility for this model which follows from the fluctuation-dissipation theorem and is given by 


$$
\chi=G_{0}(p=0)=\left(m^{2}\right)^{-1} \propto\left(T-T_{c}\right)^{-1}
$$

Thus $\gamma=1$. At $T=T_{c}$

$$
G_{0}(p) \propto \frac{1}{p^{2}}
$$

and the Fourier transform has a conspicuous scaling behaviour

$$
G_{0}(x) \propto \frac{1}{|x|^{D-2}}
$$

Therefore, $\eta=0$. The Fourier transform at non-zero $m^{2}$ is

$$
G(x)=\int \frac{d^{D} p}{(2 \pi)^{D}} \frac{e^{-i \vec{p} \cdot \vec{x}}}{p^{2}+m^{2}} .
$$

This integral is easily done for $D=3$ but is nontrivial in other dimensions. But the correlations decay exponentially with a scale $m^{-1}$ irrespective of the dimensionality $D$. Hence one gets as the correlation length

$$
\xi=m^{-1} \propto\left(T-T_{c}\right)^{-1 / 2}
$$

resulting in $v=1 / 2$. The specific heat exponent alpha is found from

$$
\begin{aligned}
C \propto & \int d^{D} x d^{D} y\left\langle\phi^{2}(x) \phi^{2}(y)\right\rangle \\
= & \int d^{D} x d^{D} y\left\langle\int \frac{d^{D} p}{(2 \pi)^{D}} \tilde{\phi}(p) e^{-i \vec{p} \cdot \vec{x}} \int \frac{d^{D} q}{(2 \pi)^{D}} \tilde{\phi}(q) e^{-i \vec{q} \cdot \vec{x}}\right. \\
& \left.\times \int \frac{d^{D} r}{(2 \pi)^{D}} \tilde{\phi}(r) e^{-i \vec{r} \cdot \vec{y}} \int \frac{d^{D} s}{(2 \pi)^{D}} \tilde{\phi}(s) e^{-i \vec{s} \cdot \vec{y}}\right\rangle_{c} \\
= & \int \frac{d^{D} p}{(2 \pi)^{D}} \int \frac{d^{D} q}{(2 \pi)^{D}}\langle\tilde{\phi}(p) \tilde{\phi}(-p) \tilde{\phi}(q) \tilde{\phi}(-q)\rangle_{c} \\
= & \int \frac{d^{D} p}{(2 \pi)^{D}}\left[G_{0}(p)\right]^{2} \\
= & \int \frac{d^{D} p}{(2 \pi)^{D}}\left[p^{2}+m^{2}\right]^{-2} \propto m^{D-4} \propto\left(T-T_{c}\right)^{-\frac{4-D}{2}}
\end{aligned}
$$

Hence $\alpha=(4-D) / 2$. The spontaneous magnetization index $\beta$ cannot be obtained within the Gaussian framework as the theory becomes unstable in the broken symmetry state. This is a first step towards resolving the complicated issue of critical exponents and universality. This theory does not yield correct exponents as the interaction term is missing in the free energy though it satisfies the scaling laws. 


\section{Appendix}

\section{Field Theoretic RG}

We briefly sketch the basic ideas of Field Theoretic RG for a scalar field. The Free energy density can be written as

$$
F=\frac{r_{0}}{2} \phi^{2}+\frac{1}{2}(\vec{\nabla} \phi)^{2}+\frac{u_{0}}{4 !} \phi^{4}
$$

We focus on the two point correlation function $\Gamma^{(2)}(k)$ at the critical point. The crucial point is that we must have a finite $\Gamma^{(2)}(k)$ as the cut-off $\Lambda$ on the momentum space variable goes to infinity (or equivalently the lattice spacing tending to zero in real space). The behaviour of $\Gamma^{(2)}(k)$ at the critical point is

$$
\Gamma^{(2)}(k) \propto k^{2-\eta}
$$

for $k \ll \Lambda$. Dimensional analysis suggests that $\Gamma^{(2)}(k)$ should have the form

$$
\Gamma^{(2)}(k)=k^{2}\left(\frac{k}{\Lambda}\right)^{-\eta}\left[1+B\left(\frac{k}{\Lambda}\right)^{\omega_{1}}+\ldots \ldots .\right]
$$

Naïve power counting yields $k^{2}$ as the dimension of $\Gamma^{(2)}(k)$ which is independent of the dimensionality of space. Since we are interested in the long-wavelength behaviour of $\Gamma^{(2)}(k)$, we would obviously like to take the limit $k \rightarrow 0$. But that also involves the limit $\Lambda \rightarrow \infty$ and therein lies the problem since it renders the leading term meaningless. To overcome the difficulty we introduce an arbitrary length scale $\mu$ in the problem and write

$$
\left(\frac{\mu}{\Lambda}\right)^{\eta} \Gamma^{(2)}(k)=k^{2}\left(\frac{k}{\mu}\right)^{-\eta}\left[1+B\left(\frac{k}{\Lambda}\right)^{\omega_{1}}+\ldots \ldots . .\right]
$$


The limit $\Lambda \rightarrow \infty$ will cause no problem on the R.H.S of Eq.(B.0.3). The L.H.S defines the renormalized vertex function

$$
\Gamma_{R}^{(2)}(k)=\left(\frac{\mu}{\Lambda}\right)^{\eta} \Gamma^{(2)}(k)=Z_{\phi}^{-1} \Gamma^{(2)}(k)
$$

We define the renormalized field as

$$
\phi_{R}(x)=Z_{\phi}^{-1 / 2} \phi(x)
$$

to obtain

$$
\left\langle\phi_{R}(x) \phi_{R}\left(x^{\prime}\right)\right\rangle=Z_{\phi}^{-1}\left\langle\phi(x) \phi\left(x^{\prime}\right)\right\rangle
$$

which remains finite as $\Lambda \rightarrow \infty$. The factor $Z_{\phi}$ is the wave-function renormalization.

To appreciate the difficulty let us calculate the correlation functions to one loop order directly by using the Free energy given in Eq.(B.0.1). To one loop order $\Gamma^{(2)}(k)$ becomes

$$
\Gamma^{(2)}(k)=k^{2}+r_{0} \frac{u_{0}}{2} \int \frac{d^{D} p}{(2 \pi)^{D}} \frac{1}{p^{2}+r_{0}} .
$$

In $D=4$ with the upper cut-off $\Lambda$ we obtain

$$
\Gamma^{(2)}(k)=k^{2}+r_{0}+\frac{u_{0}}{2}\left[\frac{\Lambda^{2}}{16 \pi^{2}}-\frac{r_{0}}{16 \pi^{2}} \ln \frac{\Lambda^{2}}{r_{0}}-\frac{1}{16 \pi^{2}} \frac{r_{0}}{\Lambda^{2}}\right]+O\left(u_{0}^{2}\right)
$$

It can be seen that the two point function does not have a well defined limit as $\Lambda \rightarrow \infty . Z_{\phi}$ cannot remove the divergence in the above limit. For the massive theory

$$
\begin{aligned}
\Gamma_{R}^{(2)}(k) & =k^{2}+\text { other terms } \\
\text { or }\left.\quad \frac{\partial \Gamma_{R}^{(2)}(k)}{\partial k^{2}}\right|_{k^{2}=0} & =1 .
\end{aligned}
$$

For a massless theory this differentiation cannot be performed at $k^{2}=0$. Thus $Z_{\phi}$ is determined from the equation

$$
\begin{aligned}
\left.Z_{\phi}^{-1} \frac{\partial \Gamma^{(2)}(k)}{\partial k^{2}}\right|_{k^{2}=0} & =1 \\
\text { or } \quad Z_{\phi} & =1+O\left(u_{0}^{2}\right)
\end{aligned}
$$

This equation predicts that there is no contribution to $Z_{\phi}$ at $O\left(u_{0}\right)$.

To restore the finiteness of the R.H.S of Eq.(B.0.6) we introduce a renormalized mass defined through the relation 


$$
\Gamma_{R}^{(2)}\left(k^{2}=0\right)=r=Z_{\phi} Z_{R}^{-1} r_{0}
$$

To one loop order we can write,

$$
Z_{r}^{-1}=1+\frac{u_{0}}{32 \pi^{2}}\left[\frac{\Lambda^{2}}{r_{0}}-\ln \frac{\Lambda^{2}}{r_{0}}-\frac{r_{0}}{\Lambda^{2}}+\ldots . .\right]
$$

The next non-trivial correlation function is the one corresponding to the four point vertex function $\Gamma^{(4)}\left(\left\{k_{i}\right\}, u_{0}, r_{0}, \Lambda\right)$ To one loop order the contribution is given by

$$
\begin{aligned}
\Gamma^{(4)}\left(\left\{k_{i}\right\}, u_{0}, r_{0}, \Lambda\right)= & -u_{0}+\frac{u_{0}^{2}}{2}\left[\int^{\Lambda} \frac{d^{D} p}{(2 \pi)^{D}} \frac{1}{\left(p^{2}+r_{0}\right)\left[\left(\vec{k}_{1}+\vec{k}_{2}-\vec{p}\right)^{2}+r_{0}\right]}\right. \\
& + \text { two permutations }]
\end{aligned}
$$

Evaluation in $D=4$ yields

$$
\begin{aligned}
\Gamma^{(4)}\left(\left\{k_{i}\right\}, u_{0}, r_{0}, \Lambda\right)= & -u_{0}+\frac{u_{0}^{2}}{2}\left[\frac{3}{16 \pi^{2}} \ln \frac{\Lambda^{2}}{r_{0}}+\mathcal{F}\left(\frac{\left(\vec{k}_{1}+\vec{k}_{2}\right)^{2}}{r_{0}}\right)\right. \\
& \left.+\mathcal{F}\left(\frac{\left(\vec{k}_{1}+\vec{k}_{3}\right)^{2}}{r_{0}}\right)+\mathcal{F}\left(\frac{\left(\vec{k}_{2}+\vec{k}_{3}\right)^{2}}{r_{0}}\right)+O\left(\frac{r_{0}}{\Lambda^{2}}, \frac{k^{2}}{\Lambda^{2}}\right)\right]
\end{aligned}
$$

where the function $\mathcal{F}(x)$ is independent of $\Lambda$. The wave function renormalization leads to

$$
\Gamma_{R}^{(4)}=Z_{\phi}^{-2} \Gamma^{(4)}=\left(1+O\left(u_{0}^{2}\right)\right) \Gamma^{(4)} .
$$

as $Z_{\phi}$ does not contain any $O\left(u_{0}\right)$ term. Now even $Z_{r}$ and $Z_{\phi}$ together cannot resolve the problem of taking the limit $\Lambda \rightarrow 0$ in the Eq.(B.0.12). Hence, one introduces yet another renormalization constant through the relation

$$
\Gamma_{R}^{(4)}\left(\left\{k_{i}\right\}=0\right)=-g
$$

This implies

$$
\begin{aligned}
-g & =-u_{0}+\frac{u_{0}^{2}}{2}\left[\frac{3}{16 \pi^{2}} \ln \frac{\Lambda^{2}}{r_{0}}-\frac{3}{16 \pi^{2}}+O\left(\frac{r_{0}}{\Lambda^{2}}\right)\right] \\
g & =u_{0}-\frac{3 u_{0}^{2}}{32 \pi^{2}}\left[\ln \frac{\Lambda^{2}}{r_{0}}-1+O\left(\frac{r_{0}}{\Lambda^{2}}\right)\right]+O\left(u_{0}^{3}\right) \\
\text { or } \quad u_{0} & =g+\frac{3 g^{2}}{32 \pi^{2}}\left[\ln \frac{\Lambda^{2}}{r_{0}}-1+O\left(\frac{r_{0}}{\Lambda^{2}}\right)\right] \\
& =Z_{\phi}^{2} Z_{u}^{-1} g
\end{aligned}
$$


Substituting $u$ in terms of $u_{R}$ in Eq.(B.0.12)

$$
\begin{aligned}
\Gamma^{(4)}\left(\left\{k_{i}\right\}, g, r, \Lambda\right)= & -g-\frac{g^{2}}{32 \pi^{2}}\left[\mathcal{F}\left(\frac{\left(\vec{k}_{1}+\vec{k}_{2}\right)^{2}}{r_{0}}\right)+\mathcal{F}\left(\frac{\left(\vec{k}_{1}+\vec{k}_{3}\right)^{2}}{r_{0}}\right)\right. \\
& \left.+\mathcal{F}\left(\frac{\left(\vec{k}_{2}+\vec{k}_{3}\right)^{2}}{r_{0}}\right)+\frac{3}{16 \pi^{2}}+O\left(\frac{k^{2}}{r}\right)\right]
\end{aligned}
$$

Now if we take the limit of $\Lambda \rightarrow \infty$, the result is finite. $Z_{u}$ is the coupling constant renormalization. This makes the two point and four point correlation functions finite in this limit. It now remains to be shown that this method will automatically make the higher order correlation functions finite.

To do that, we consider the general interaction of the form $\phi^{r}$ and a graph with $E$ external legs. The number of internal lines $I$ is given by

$$
I=\frac{1}{2}(r n-E)
$$

where $n$ is the number of times the interaction acts. Of the $r n$ lines it produces, $E$ are joined to the external legs. The remaining $r n-E$ lines have to be paired which gives rise to the Eq.(B.0.17). The degree of primitive divergence $\delta$ of a graph with $l$ loops and $n$ interaction vertices is

$$
\delta=l D-2 I
$$

where there is no divergence due to subintegrations. We now relate $l$ to the number of internal lines $I$. For each internal line there is an associated momentum, of which not all are independent. At each of the $n$ interaction vertices there is a momentum conserving delta function. But one of the conservation laws corresponds to the overall momentum conservation and thus only $I-(n-1)$ are independent. So,

$$
l=I-(n-1) \text {. }
$$

Thus, the degree of divergence becomes,

$$
\delta=\left(\frac{r D}{2}-r-D\right) n+D+E-\frac{D E}{2}
$$

The above formula has a n-dependent part. For a given $r$, there is a choice of $D$ for which the coefficient of $n$ vanishes. This is the critical dimension $D_{c}$ given by

$$
D_{c}=\frac{2 r}{r-2} \text {. }
$$

Clearly, for $D=D_{c}, \delta$ is independent of the number of loops. For $D>D_{c}, \delta$ increases with the number of loops and it would be impossible to absorb all the divergences by introducing a finite number divergences. For $D \leq D_{c}$, it is possible to absorb all the divergences in a finite number of renormalization constants 
rendering a finite theory in the limit of $\Lambda \rightarrow 0$. The theory then becomes renormalizable for $D=D_{c}$, Super-renormalizable for $D<D_{c}$ and non-renormalizable for $D>D_{c}$. For the quartic interaction $r=4$ and $D_{c}=4$. Thus $\delta$ in $D=4$ in $\phi^{4}$ theory is independent of the number of loops. It is clear from Eq.(B.0.20) that only primitive divergence comes from $E \leq 4$ and thus once the $\Gamma^{(2)}$ and $\Gamma^{(4)}$ are rendered finite in the infinite cut-off limit. For the massive $\phi^{4}$ theory, the $\Gamma^{(2)}$ and $\Gamma^{(4)}$ are rendered finite if

$$
\begin{gathered}
\left.\frac{\delta \Gamma_{R}^{(2)}}{\delta k^{2}}\right|_{k=0}=1 \\
\Gamma_{R}^{(2)}(k=0)=r \\
\Gamma_{R}^{(4)}\left(\left\{k_{i}\right\}=0\right)=-g
\end{gathered}
$$

For the massless theory these renormalization conditions have to change because of the presence of the anomalous dimension index $\eta$. Therefore we must evaluate derivative at $k^{2}=\mu^{2}$ and accordingly the first of Eq.(B.0.22) is changed to

$$
\left.\frac{\delta \Gamma_{R}^{(2)}}{\delta k^{2}}\right|_{k^{2}=\mu^{2}}=1 .
$$

Similarly, The condition of second of Eq.(B.0.22) is to be modified to ensure that $\Gamma_{R}^{(2)}(k=0)=0$. Finally, we focus on the four point correlation function where it is clear from Eq.(B.0.11) that for $r_{0}=0$ we do not have a well defined integral when the external momentum vanishes. Hence the condition on $\Gamma_{R}^{(4)}\left(\left\{k_{i}\right\}\right)$ has to be changed to a condition at finite momentum and we choose the symmetric point $\mu_{S}$, where

$$
k_{1}^{2}=k_{2}^{2}=k_{3}^{2}=k_{4}^{2}=\mu^{2}
$$

Since

$$
\vec{k}_{1}+\vec{k}_{2}+\vec{k}_{3}+\vec{k}_{4}=0
$$

because of overall momentum conservation, we have

$$
0=\sum_{i} k_{i}^{2}=4 \mu^{2}+12\left(\vec{k}_{i} \cdot \vec{k}_{j}\right)
$$

or

$$
\vec{k}_{i} \cdot \vec{k}_{j}=-\frac{\mu^{2}}{3} \quad \text { for } \quad i \neq j
$$

For $i=j k_{i}^{2}=\mu^{2}$ and thus for $\mu_{S}$

$$
\vec{k}_{i} \cdot \vec{k}_{j}=\frac{\mu^{2}}{3}\left(4 \delta_{i j}-1\right)
$$


Thus the renormalization conditions for the massless theory are

$$
\begin{aligned}
\left.\frac{\delta \Gamma_{R}^{(2)}}{\delta k^{2}}\right|_{k^{2}=\mu^{2}} & =1 \\
\Gamma_{R}^{(2)}(k=0) & =0 \\
\Gamma_{R}^{(4)}\left(\mu_{S}\right) & =-g .
\end{aligned}
$$

To end this discussion, we point out an apparent contradiction. The field theoretic tools we have developed so far are supposed to deal with critical phenomena. However, critical phenomena is uncomplicated for $D>4$ and is non-trivial for $D<$ 4 , while the process of renormalization shows that the theory is non-renomalizable for $D>4$ and super-renormalizable for $D<4$. If this appears to be a contradiction, we must remember that the question of renormalizability just raised involves an ultraviolet cut-off, while for the critical phenomena the relevant behaviour is in the infrared region. To make things clear, we consider the integral appearing in $\Gamma^{(4)}(k=0)$

$$
I(m)=\int_{0}^{\Lambda} \frac{d^{D} p}{(2 \pi)^{D}}\left(\frac{1}{p^{2}+m^{2}}\right)^{2}
$$

First, we demonstrate that this integral can be made ultraviolet finite by successive partial integrations involving the 't Hooft and Veltman technique. We note that

$$
\frac{1}{D} \sum_{i=1}^{D} \frac{\partial}{\partial p_{i}}=1
$$

and insert it in Eq.(B.0.26) to obtain

$$
\begin{aligned}
I(m)= & \sum_{i=1}^{D} \frac{1}{D} \int_{0}^{\Lambda} \frac{\partial}{\partial p_{i}} \frac{p_{i}}{\left(p^{2}+m^{2}\right)^{2}} \frac{d^{D} p}{(2 \pi)^{D}} \\
& +\sum_{i=1}^{D} \frac{4}{D} \int_{0}^{\Lambda} \frac{p_{i} p_{i}}{\left(p^{2}+m^{2}\right)^{3}} \frac{d^{D} p}{(2 \pi)^{D}}
\end{aligned}
$$

but

$$
\int_{0}^{\Lambda} \frac{p_{i}}{\left(p^{2}+m^{2}\right)^{2}} \frac{d^{D} p}{(2 \pi)^{D}}=0,
$$

so

$$
\begin{aligned}
I(m) & =\frac{4}{D} I(m)-\frac{4}{D} m^{2} \int \frac{d^{D} p}{(2 \pi)^{D}} \frac{1}{\left(p^{2}+m^{2}\right)^{3}} \\
\text { or } \quad I(m)\left[1-\frac{4}{D}\right] & =-\frac{4 m^{2}}{D} \int \frac{d^{D} p}{(2 \pi)^{D}} \frac{1}{\left(p^{2}+m^{2}\right)^{3}}
\end{aligned}
$$


The integral on the R.H.S of Eq.(B.0.29) is ultraviolet convergent for $D<6$. We can now take the limit $\Lambda \rightarrow \infty$ for $D<6$ and hence $I(m)$ scales as $m^{D-4}$. The integral diverges for $D<4$ as $m \rightarrow 0$ which is the problem of critical phenomena - the infrared divergence. It is worthwhile to note that as the integral is made ultraviolet finite by successive partial- $p$ 's, the scaling behaviour w.r.t $m$ is exactly the simple power count $m^{\delta}$. Thus the infrared behaviour of the loop integrals is $m^{\delta}$ with $\delta$ given by Eq.(B.0.20). If we consider the second term of Eq.(B.0.20), then we find that it is the term associated with the free field theory. The free field will dominate i.e. give the correct infrared singularity if the first term is positive. The first term is positive if $D>D_{c}$ and thus for the infrared question, it is the free field theory which is relevant for $D>D_{c}$, whereas for $D<D_{c}$, the infrared behaviour is changed as the first term lowers the values of $\delta$ and one observes non-trivial critical singularities. 


\section{Index}

A

advection of a passive scalar 252

Allen and Cahn result 162

angular momentum 103

anisotropic correlation function 44 anomalous dimension exponent 71 anomaly in limits 248

\section{B}

backflow effect 284

ballistic deposition 185

bath variables 27

beam intensity 48

binary alloy 104

binary fluid mixture 104

Burger's equation 54

\section{C}

chaotic evolution 236

characteristic length of defect 165

chemical potential 48,133

coherent structure 54

coloured noise 29

complex sound speed 130 concentration gradient 104

conservation law 39

consolute point 131

constant volume specific heat 129

correction-to-scaling 142

correlation

function 16

length 58

coupling constants 58

coupled growth models 227

Curie

point 2

temperature 2

\section{D}

Das Sarma-Tamborenea model 214

defect core 164

density fluctuations 127

depinning transition 224

diagrammatic representation 88

diffusive mode

dimensional analysis 53

discrete model for surface growth 214

dissipation range 241

dissipative terms 29

domain wall 164

dominance of critical fluctuations 123

driven diffusive equation 250 
droplet size 162

dynamic

renormalization group 65

scaling exponent 65

dynamical systems 268

\section{E}

Edwards-Wilkinson model 48, 188 effective equation of motion 74

energy

spectrum 53

transport 52

enstrophy 239

entropy fluctuations 133

equations of motion in a binary fluid 109

Ertas and Kardar model 227

evaporation dynamics 48

external electric field 151

F

$f(\alpha)$ curve 257

ferromagnetic system 73,38

field theoretic form 79

finite dissipation 232

flow around a cylinder 231

fluctuation dissipation

relation 44

theorem 85

Fokker-Planck equation 35

Fourier space 290

fractal dimension 257

free energy functional 12

frequency dependent specific heat 130

functional integral 287

\section{G}

Galilean invariance 195

Gaussian distribution 31

model 60

model for polymers 277

tail 171

Ginzburg-Landau free energy 12

Goldstone mode 135

growth law for domains 163

growth model with

correlated noise 217

nonlocality 219

surface diffusion 207

H

Heisenberg ferromagnet 73

high frequency limit 97

Hopf bifurcation 231

I

ideal chain 278

incompressible flow 50, 230

inertial range 52, 234

intermittency 53, 255

internal 'colour' index 98

inverse correlation length 37

inviscid limit 239

irrelevant variables 62

isentropic process 131

Ising spin 44

isothermal susceptibility 2

J

Jacobian 80

K

Kadanoff construction 57

Kardar-Parisi-Zhang equation 49, 194

Katz, Lebowitz and Spohn model 43

Kawasaki function 127 
Kolmogorov phenomenology 52, 237

Kosterlitz-Thouless flow 223

Kraichnan's method 98

Kramers-Kronig relation 85, 148

L

lambda transition 132

Landau's observation 255

Langevin equation

for polymer dynamics 279

late stage behaviour 179

lateral correlation 187

leading $\log 207$

liquid gas critical point 10

local

curvature driven growth 208

field 6

magnetization 6

longrange interaction 277

Lyapunov exponent 236

\section{M}

Maxwell-Boltzmann distribution 28

Mazenko's approach 176

Menevau and Sreenivasan's exponent 260 mesoscopic quantum wave function 132 miscibility 39

mode coupling theories 83

for KPZ 203

models A, B, J, H 38, 40

models of growth 47

molecular beam epitaxy 215

Mukherji and Bhattacharjee's modification 219

multiscaling 174

multifractal distribution 257

\section{N}

Navier-Stokes equation 229

n-component model 136 noise 29

nonlinear transformation 174

normal

fluid 132

modes 280

numerical work of Hayot and Jayaprakash 219

\section{0}

Obukhov and Kolmogorov phenomenology 255

Ohta, Jasnow and Kawasaki method 175

Onsager coefficient 79

order parameter profile 159

$\mathbf{p}$

partition function 80

periodic terms 222

perturbation theory

diagrammatics 117

poles in $\mathrm{G}(\mathrm{k}, \omega) 114$

polymer chain 275

Porod's law 169

Prandtl's mixing length hypothesis 245

\section{a}

quasi-Lagrangian approach 265

quenched noise 224

R

random force 29

randomly stirred model 246

recursion relations 62,201

renormalization group 57, 179, 196, 265

response to a weak force 146

reversible terms 73

Reynold's number 230

roughening transition 221

roughness exponent 193

Rouse model 283 
S

scaling Ansatz 123

second sound 135

self consistent

mode-coupling 86

perturbation theory 263

self energy $71,78,117$

shear viscosity 105

shell model 270

singular lines 45

slow variables 60

sound propagation 129

spherical limit 98, 172

steepest descent 173

Stokes' law 105

structure factor 171

supercurrent 133

superfluid transition 132

surface diffusion 207

surface tension 193

\section{$T$}

Taylor's hypothesis 265

thermal conductivity 105

three point function 155, 242

topological defect 164

turbulence 50, 229 turbulent state 229

two dimensional Ising model 57

U

upper critical dimension 202

V

Van der Waal's equation 9

vortex line 164

W

wavelet basis 270

weak scaling 227

weak solution 233

white noise 30

Wick's theorem 113

Wolf-Villain model 215

Z

zero temperature fixed point 182 